\title{
THE ADDITIVE STRUCTURE OF INTEGER GROUPS AND P-ADIC NUMBER FIELDS
}

\author{
LEWIS ROBERTSON ${ }^{1}$ AND BERT M. SCHREIBER ${ }^{2}$
}

A locally compact abelian group is said to be a topological $p$-group if the closed subgroup generated by any element is isomorphic to either the $p$-adic integers or a finite $p$-group which is a quotient of the $p$-adic integers. Braconnier has shown that any totally disconnected abelian group with totally disconnected dual is isomorphic to a local direct product of topological p-groups. (See $[1$, p. 49], $[4,(6.16)]$, and also [9, 3.1 and 3.21].) This is one instance of the generalization that every locally compact abelian group is in some sense built up out of pieces which are either discrete groups, connected groups, or else groups which are related to $p$-adic integers and $p$-adic number fields. Various structure-theoretic results give other concrete examples of the validity of this generalization, in particular the results on monothetic groups [4, (25.16) and (25.17)], torsion-free compact groups $[4,(25.8)]$, torsion-free divisible groups $([6$, p. 940$]$ and $[4$, (25.33)]), and other "natural" classes of groups [7, p. 189], [8, Theorems 2.1 and 2.10$],[9,3.17,3.27,4.5,4.13]$. All this indicates the need for a purely structure-theoretic approach to the study of $p$-adic integers and number fields, and a clarification of the special properties of these important groups. We obtain below some characterizations of the ordinary integers, $p$-adic integers, $p$-adic number fields, the line, the circle, and $\boldsymbol{Z}_{\boldsymbol{p}}^{\infty}$, as those groups which satisfy certain conditions on their closed subgroups.

1. Definition. Let $G$ be a L.C.A. group. $G$ is said to be almost finite (resp., almost cyclic, almost compact, or almost discrete) if every proper closed subgroup of $G$ is finite (resp., of finite index, compact, or open). Note that $G$ is almost finite (resp., almost compact) iff the character group $\hat{G}$ of $G$ is almost cyclic (resp., almost discrete). $G$ is said to be an integer group if $G$ is infinite and almost cyclic.

$T$ denotes the circle, and $Z, R, F_{p}, J_{p}$ denote the additive structures of the integers, line, $p$-adic number field, and $p$-adic integers, respectively. (See $[4,(10.3)$ and $(10.10)]$ for details on $F_{p}$ and $J_{p}$.) We will also make use of the discrete groups $Z_{p}^{\infty}[4$, p. 3]

Received by the editors July 21, 1967.

${ }^{1}$ Supported in part by NSF grant GP-6944.

2 Supported in part by NSF grant GP-7832. 
2. Proposition. A L.C.A. group is almost cyclic iff it is $\boldsymbol{Z}, J_{\boldsymbol{p}}$, or finite.

Proof. Finite groups clearly satisfy all of the conditions at the beginning of Definition 1 . Let $G$ be infinite and almost cyclic. $G$ cannot contain a closed subgroup topologically isomorphic to $R$, so $G$ contains a compact open subgroup by the fundamental structure theorem $[4,(24.30)]$. Since $G$ is almost cyclic, we see that $G$ is either compact or discrete.

Suppose $G$ is discrete, so that every proper subgroup of $G$ has finite index. If $0 \neq x \in G$, then in particular the subgroup of $G$ generated by $x$ has finite index in $G$. Hence $G$ is finitely generated and is a direct product of cyclic groups. But if $G$ can be decomposed as a direct product of two nontrivial subgroups, then one of them is infinite and the other is a proper subgroup of infinite index, which is impossible. Thus $G=\boldsymbol{Z}$.

Now suppose $G$ is compact. Then $\hat{G}$ is a discrete group in which every proper subgroup is finite. Hence $\hat{G}=\boldsymbol{Z}_{p}^{\infty}\left[\mathbf{5}\right.$, p. 22], and $G=J_{P}$, $[4,(25.2)]$, for some prime $p$.

3. CoROllary. $G$ is an integer group iff $G$ is the ordinary integers $\boldsymbol{Z}$ or the p-adic integers $J_{p}$.

4. Corollary. $G$ is infinite and almost finite iff $G=\boldsymbol{T}$ or $G=Z_{p}^{\infty}$.

5. Definition. A L.C.A. group $G$ is said to be relatively discrete if every proper closed subgroup is a discrete group.

6. Proposition. $G$ is nondiscrete and relatively discrete iff $G=T$, or $G=R$.

Proof. By the fundamental structure theorem, either $G$ contains $R$ as a closed subgroup, or $G$ has a compact open subgroup. It follows that $G=R$ or $G$ is compact. But if $G$ is compact, then it is almost finite and hence equal to $T$ by Corollary 4 .

7. Proposition. $G$ is nondiscrete and almost discrete iff $G=J_{p}$ or $G=F_{p}$.

Proof. As in Proposition 2, $G$ contains a compact open subgroup $K$, which is then clearly almost discrete also. Since every open subgroup of $K$ has finite index in $K, K$ is almost cyclic. By 2, $K$ is topologically isomorphic to $J_{p}$ for some prime $p$. Since $F_{p}$ is divisible, the identification of $K$ with $J_{P}$ can be extended to a homomorphism $\phi$ of $G$ onto an open subgroup of $F_{p}$. $\phi$ is continuous and open because it is a homeomorphism of the open subgroup $K$ of $G$ onto the open sub- 
group $J_{p}$ of $F_{p}$. If $\operatorname{ker} \phi \neq\{0\}$, then $\operatorname{ker} \phi$ is open because $G$ is almost discrete. Hence ker $\phi \cap K \neq\{0\}$, contradicting the definition of $\phi$. Thus $\phi$ is a topological isomorphism, and the proposition follows from $[4,(10.16)]$.

Proposition 7 yields a characterization of the $p$-adic number fields $F_{p}$ as the only noncompact nondiscrete groups which are almost discrete. As an application of this characterization, we give a purely structure-theoretic proof that $F_{p}$ is self-dual [4, (25.1)].

8. Proposition. Let $G$ denote the dual group of $F_{p}$. Then $G=F_{p}$.

Proof. $G$ is noncompact and nondiscrete. $F_{p}$ is almost compact $[4,10.16]$, and so $G$ is almost discrete. Thus it follows from Proposition 7 that $G=F_{q}$ for some prime $q$, and we need only show that $p=q$. This may be seen by noting that $G$ contains an open subgroup isomorphic to $J_{p}$ because $F_{p} / J_{p}=Z_{p}^{\infty}$, or, alternatively, one may use $[9,3.18]$.

9. Using Propositions 7 and 8 together, we have the following corollary.

Corollary. $G$ is noncompact and almost compact iff $G=F_{p}$ or $G=Z_{p}^{\infty}$. In particular, the groups $\boldsymbol{F}_{p}$ are the only noncompact nondiscrete L.C.A. groups such that every proper closed subgroup is compact and open.

10. We summarize our results in the proposition below, using the word group to mean locally compact abelian topological group.

Proposition. The properties listed in Definitions 1 and 5, together with appropriate combinations of the properties (a) discrete, (b) compact, (c) nondiscrete, and (d) noncompact, yield group-theoretic characterizations of $\boldsymbol{Z}, J_{p}, F_{p}, R, T$, and $\boldsymbol{Z}_{p}^{\infty}$.

11. REMARK. It is possible to arrive at the $p$-adic number fields by standard constructions which are valid for arbitrary groups, and at the same time gain some insight into why it is natural for these groups to be endowed with a ring-theoretic structure. One can start with the $p$-element cyclic group $\boldsymbol{Z}_{\boldsymbol{p}}$, and define $\boldsymbol{Z}_{\boldsymbol{p}}^{\infty}$ to be the minimal divisible extension of $Z_{p}$ [4, (A.15)]. $J_{p}$ can then be defined as the dual of $\boldsymbol{Z}_{p}^{\infty}$, and the fact that $\boldsymbol{Z}_{\boldsymbol{p}}^{\infty}$ can be realized as a subgroup of the circle immediately provides $J_{p}$ with a ring-theoretic structure, since the character group of $\boldsymbol{Z}_{p}^{\infty}$ is precisely the same set (with the same additive structure) as the ring of endomorphisms of $\boldsymbol{Z}_{p}^{\infty}$. Proceeding along these lines, $F_{p}$ can be defined as the minimal divisible extension of $J_{p}$, and it is not hard to show that $\boldsymbol{F}_{p}$ becomes a ring as a conse- 
quence of $J_{p}$ being a ring. All of the significant results on $F_{p}$ can be deduced as easily from these definitions as any others, provided that one has available the standard theorems on minimal divisible extensions and duals of subgroups and quotient groups. One of the few advantages of the usual infinite expansion notation is that it provides a very clear picture of the topology of $F_{p}$ as a weak direct product and strong direct product of copies of $\boldsymbol{Z}_{\boldsymbol{p}}$.

12. REMARK. The program of obtaining information about a group from a knowledge of its closed subgroups is developed in [8] and [10]. Structure-theoretic characterizations of $\boldsymbol{Z}$ and $\boldsymbol{R}$ (resp., $\boldsymbol{T}, \boldsymbol{R}, J_{\boldsymbol{p}}$, and $\left.F_{p}\right)$ can be obtained from $[2$, Theorems $1,2,3],[3$, p. 141], and [11, Theorem 5.2, p. 486] (resp., [8, Theorem 2.1, p. 23]).

\section{REFERENCES}

1. J. Braconnier, Sur les groupes topologiques localment compacts, J. Math. Pures Appl. 27 (1948), 1-85.

2. H. Chu, A characterization of integer groups and real groups, Duke Math. J. 28 (1961), 125-132.

3. - Bonded groups, J. Math. Soc. Japan 13 (1961), 131-143.

4. E. Hewitt and K. Ross, Abstract harmonic analysis. I, Springer-Verlag, Berlin, 1963.

5. I. Kaplansky, Infinite Abelian groups, Univ. of Michigan Press, Ann Arbor, 1954.

6. G. W. Mackey, A remark on locally compact abelian groups, Bull. Amer. Math. Soc. 52 (1946), 940-944.

7. L. S. Pontryagin, Topologische Gruppen. I, II, 2nd ed., Teubner, Leipzig, 19571958.

8. N. Rickert, Locally compact topologies for groups, Trans. Amer. Math. Soc. 126 (1967), 225-235.

9. L. Robertson, Connectivity, divisibility, and torsion, Trans. Amer. Math. Soc. 128(1967), 482-505.

10. K. Ross, Closed subgroups of locally compact abelian groups, Fund. Math. $\mathbf{5 6}$ (1964), 241-244.

11. F. Wright, Topological abelian groups, Amer. J. Math. 79 (1957), 477-496.

UNIVERSITY OF WASHINGTON 